\title{
Perioperative changes in pro and anticoagulant factors in prostate cancer patients undergoing laparoscopic and robotic radical prostatectomy with different anaesthetic techniques
}

\author{
Maria Sofra ${ }^{1}$, Anna Antenucci ${ }^{2}$, Michele Gallucci ${ }^{3}$, Chiara Mandoj ${ }^{2}$, Rocco Papalia ${ }^{3}$, Claudia Claroni ${ }^{1}$, \\ Ilaria Monteferrante ${ }^{1}$, Giulia Torregiani ${ }^{1}$, Valeria Gianaroli ${ }^{1}$, Isabella Sperdutii ${ }^{4}$ Luigi Tomao ${ }^{2}$ and Ester Forastiere ${ }^{1 *}$
}

\begin{abstract}
Background: Laparoscopic prostatectomy (LRP) may activate clotting system influencing the risk of perioperative thrombosis in patients with prostate cancer. Moreover, different anaesthetic techniques can also modify coagulant factors. Thus, the aim of this study was to investigate the effects on pro- and anti-coagulant and fibrinolytic factors of two established types of anaesthesia in patients with prostate cancer undergoing elective LRP.

Methods: 102 patients with primary prostate cancer, who underwent conventional LRP or robot-assisted laparoscopic prostatectomy (RALP), were studied and divided into 2 groups to receive total intravenous anesthesia with target-controlled infusion (TIVA-TCI) or balanced inhalation anaesthesia (BAL) prior to surgery. Before the induction of anaesthesia (T0), $1 \mathrm{hr}$ (T1) and 24 hrs post-surgery (T2), some pro-coagulant factors, fibronolysis markers, p-selectin and haemostatic system inhibitors were evaluated.

Results: Both TIVA-TCI and BAL patients showed a marked and significant increase in pro-coagulant factors and consequent reduction in haemostatic system inhibitors in the early post operative period ( $p \leq 0.004$ for each markers). Use of RALP showed a significant increase in prothrombotic markers as compared to LRP. In TIVA patients undergoing LRP, a significant reduction of $p$-selectin levels between T0 and T2 $(p=0.001)$ was observed as compared to BAL, suggesting a better protective effect on platelet activation of anaesthetic agents used for TIVA.
\end{abstract}

Conclusions: Both anaesthetic techniques significantly seem to increase the risk of thrombosis in prostate cancer patients undergoing LRP, mainly when the robotic device was utilized, encouraging the use of a peri-operative thromboembolic prophylaxis in these patients.

Keywords: TIVA-TCl anaesthesia, BAL anaesthesia, Thrombotic factors, Prostate cancer, Prostatectomy

\section{Background}

Several epidemiological studies have shown that a strong correlation exists between cancer and haemostatic system [1-4]. The interaction between cancer and the coagulation system perturbs and stimulates pro-coagulant activity, consequently inducing a pro-thrombotic state [5] and increasing the risk of thromboembolic disease (TED) [6]. Interestingly in cancer patients a systemic activation

\footnotetext{
* Correspondence: forastiere@ifo.it

'Department of Anaesthesiology, Regina Elena, Roma National Cancer Institute, Via Elio Chianesi 53, Roma 00144, Italy

Full list of author information is available at the end of the article
}

of blood coagulation has frequently been observed even in the absence of TED [2,7].

Cancer cells can activate the clotting system directly, thereby generating thrombin, or indirectly by stimulating mononuclear cells, platelets and endothelial cells to synthesize and express a variety of procoagulants [8]. The consequent formation of a fibrin matrix appears to promote tumor growth by favoring neoangiogenesis and shielding tumor cells against attack from immunocompetent cells [5]. Thrombin also works as a potent promoter of cancer growth and spread via an increase in tumor cell adhesion [9]. Some biomarkers have been 
specifically investigated for their capacity to predict TED during the course of cancer disease. Associations between elevated levels and future TED have been found for D-Dimer, prothrombin fragment $1+2(\mathrm{~F} 1+2)$, thrombinantithrombin complexes (TAT), plasminogen activator inhibitor type 1 (PAI-1), clotting factor VIII (FVIII) and soluble P-selectin [10]. These markers, not sufficiently validated in patients undergoing different intraoperative anaesthetic regimens, reflect different steps of the coagulation cascade (Figure 1). In particular, F1 +2 is released when activated factor $\mathrm{X}$ cleaves prothrombin into active thrombin and the fragment formation is a key event in the coagulation cascade. The formation of TAT complexes represents an indirect measure for the activation of the coagulatory system, because is the first amount of thrombin that binds to antithrombin (AT). Elevated FVIII levels are a well-established risk factor for first manifestation and for recurrence of TED. PAI-1 is a potent inhibitor of the fibrinolytic system while d-dimer is a stable end product of fibrin degradation and is elevated by enhanced fibrin formation and fibrinolysis [10-12]. P-selectin, a member of cell adhesion molecules, is released from the $\alpha$-granules of activated platelets and from Weibel-Palade bodies of endothelial cells. P-selectin plays a crucial role in thrombogenesis and induces a prothrombotic state by the adhesion of platelets and leukocytes to cancer cells. Levels of soluble P-selectin are elevated in patients with acute TED [13].

Surgical tissue trauma also leads to an increased risk of TED [14] even though the incidence of TED is closely related to the organ involved. The tumor sites most at risk of developing TED seem to be the pancreas, brain, and stomach [14]. In patients with advanced prostate cancers, the incidence of TED is controversial, ranging from $0.5 \%$ to $40 \%$ in the first month after surgery [3,15-17]. The increased risk of TED in prostate cancer patients undergoing radical prostatectomy recommends administering a pharmacologic anti-thrombotic prophylaxis [18-22], though the latter may cause an increase in intra-operative bleeding $[23,24]$.

To date, factors influencing the risk of perioperative thrombosis in patients undergoing prostate cancer surgery have not been identified yet. At present, we do not know whether, in addition to the risk factors already known, the use of different techniques of anesthesia may increase the risk of thrombosis in cancer patients undergoing surgery. Therefore, the main aim of this prospective study was to investigate changes in the markers most sensitive to detecting activation of the haemostatic system in patients with prostate cancer undergoing elective laparoscopic prostatectomy with two different intra-operative anaesthetic regimens, target-controlled infusion (TIVA-TCI) and balanced inhalation anaesthesia (BAL). A secondary aim was to evaluate whether using a robot device in the laparoscopic prostatectomy influences the effect of different anesthetic techniques applied.

\section{Methods}

\section{Patient population}

Between October 2009 and June 2012, 400 consecutive patients with primary prostate cancer, undergoing general anaesthesia and conventional laparoscopic radical

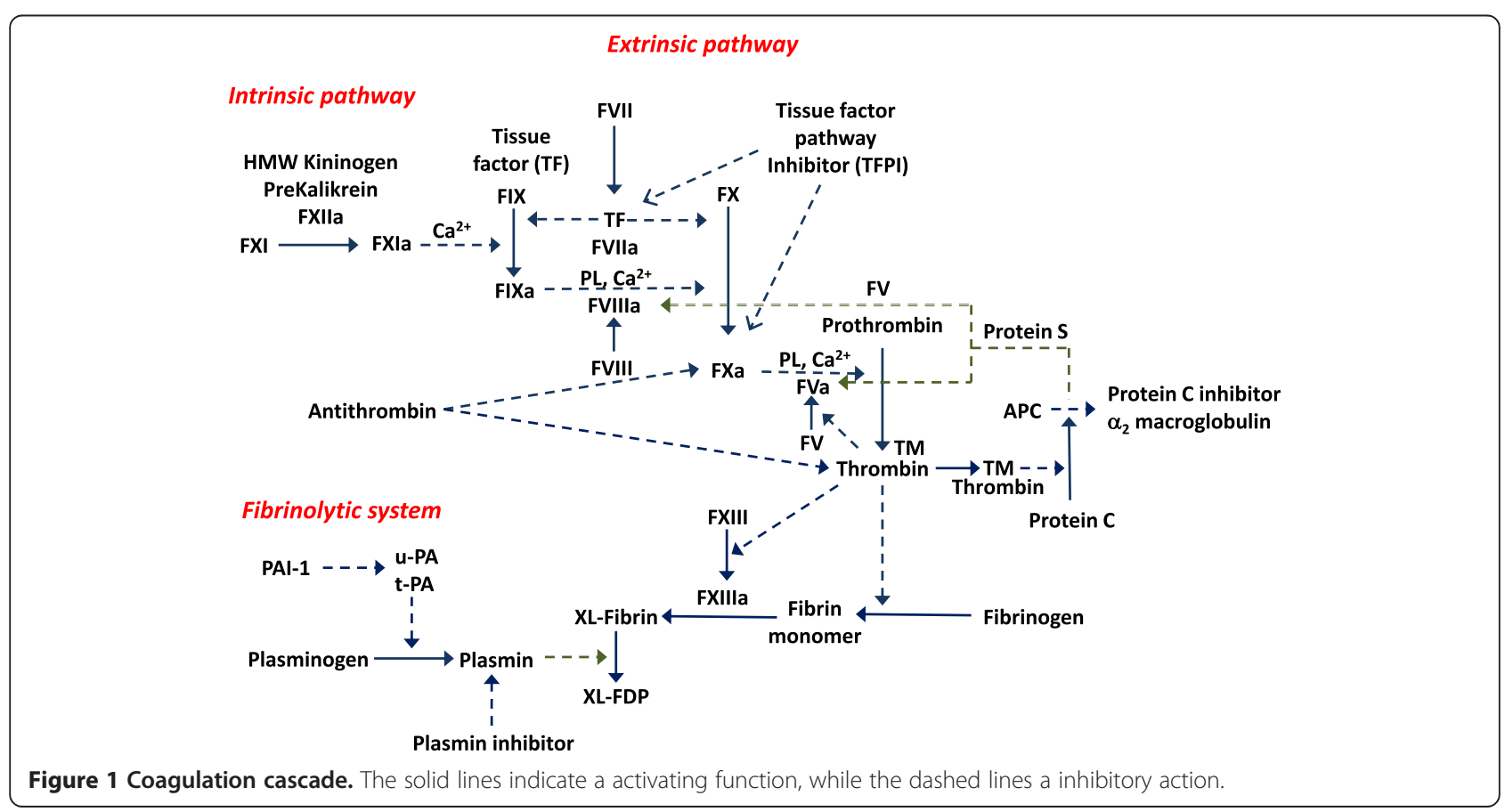


prostatectomy (LRP) or robot-assisted laparoscopic prostatectomy (RALP), were considered eligible for the study (Figure 2). This study was approved by the Ethics Committee of the Regina Elena National Cancer Institute, Rome (Prot.CE/550), and a written informed patient consent was obtained from all participants. Protocol was registered in Clinical trials.gov (NCT01998685). The inclusion criteria for the study were a newly diagnosed cancer of the prostate with histological Gleason score evaluation. Exclusion criteria included: (a) ASA $>2$, (b) metabolic equivalent task $<4$, (c) BMI $>30$, (d) no preoperative pharmacological thromboprophylaxis and/or anti-coagulant therapy, (e) history of abnormal bleeding, or abnormal coagulant factors, (f) sepsis within the last 2 weeks, (g) previous new adjuvant treatments (chemo, hormone, and radiotherapy), (h) non-steroid, anti-inflammatory and statin drugs for at least 2 wks before surgery, (i) venous or arterial thromboembolism within the last 3 months, peripheral venous disease, (l) neurological disease with extremity paresis, $(\mathrm{m})$ chronic liver disease, (n) pre-operative haemoglobin concentration $<9 \mathrm{mg} \mathrm{dl}^{-1}$, (o) prolonged duration of surgery ( $>3 \mathrm{hrs}$ ); (p) peri-operative blood transfusion, (q) inadequate material for laboratory testing. One exclusion criterion sufficed exclusion.

Out of the 400 patients with primary prostate cancer who underwent laparoscopic prostatectomy, 244 were excluded from the study for the following reasons: 218 for ASA $\geq 3,4$ for previous new adjuvant treatments, 22 for anti-inflammatory and statin therapy before surgery.

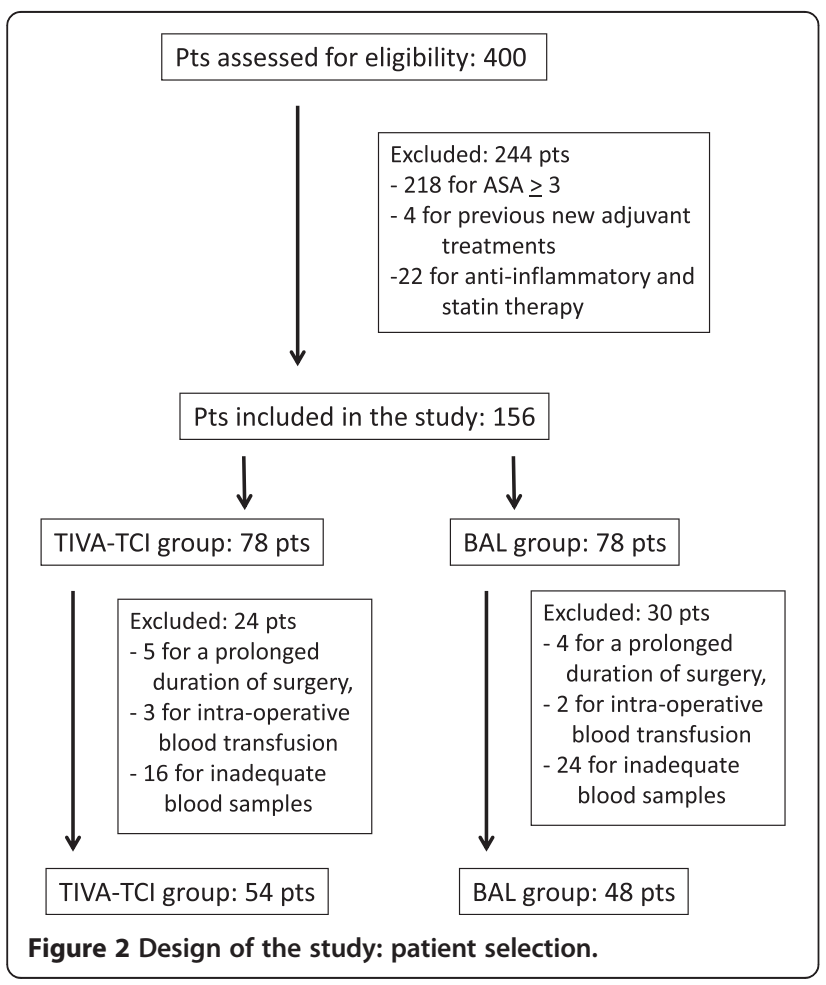

Thus, 156 patients with primary prostate cancer constituted the patient population of this randomized study and were alternatively divided into 2 groups to receive TIVA-TCI or BAL anaesthesia prior to surgery. Then, a further 54 patients were excluded: 9 for a prolonged duration of surgery, 5 for intra-operative blood transfusion and 40 for inadequate blood samples. Finally, 102 patients with primary prostate cancer comprised the patient population of the study: 54 received TIVA-TCI and 48 BAL anesthesia prior to surgery.

All patients with high-risk prostate cancer (according to Guidelines on Prostate Cancer of European Association of Urology, 2012) underwent LRP with extended pelvic lymph node dissection. Patients with intermediate risk underwent LRP or RALP .

\section{Anesthetic protocol}

The patients did not receive premedication. In the TIVA-TCI group, anaesthesia was induced with propofol (Diprivan $^{\mathrm{TM}}$, ASTRA-Zeneca, Milano, Italy) $6 \mu \mathrm{g} \mathrm{m}^{-1}$ and remifentanyl (Ultiva ${ }^{\mathrm{TM}}$, GlaxoSmith-Kline $\mathrm{AB}$, Verona, Italy) $0.4-1 \mu \mathrm{g} \mathrm{kg}^{-1} \mathrm{~min}$, simultaneously administered using two separate modules of a continuous computer-assisted TCI system. Anaesthesia was maintained with propofol $4 \mu \mathrm{g} \mathrm{ml}^{-1}$ and remifentanil $0.25 \mu \mathrm{g} \mathrm{Kg}^{-1} \mathrm{~min}$. This infusion was modified by $0.05 \mu \mathrm{g} \mathrm{kg}^{-1}$ min steps according to analgesic needs. In the BAL group, anaesthesia was induced with midazolam (Hameln pharmaceuticals Gmbh, Hameln, Germany) $0.1 \mathrm{mg} \mathrm{kg}^{-1}$ and fentanyl (Fentanest ${ }^{\mathrm{TM}}$, Pftzer, Latina, Italy) $1.5 \mu \mathrm{g} \mathrm{kg}^{-1}$ Anaesthesia was maintained with sevoflurane (Sevorane ${ }^{\mathrm{TM}}$, Abbott, Latina, Italy) $2.0 \%$, oxygen $40 \%$ and air $70 \%$ with positive pressure ventilation in a circle system, in order to achieve normocapnia.

In both groups, cisatracurium besylate Nimbex $^{\mathrm{TM}}$, Glaxo Smith Kline) $0.1-0.5 \mathrm{mg} \mathrm{kg}^{-1}$ was given to facilitate orotracheal intubation with a cuffed tube, followed by the continuous application of 0.06-0.12 $\mathrm{mg} \mathrm{kg}^{-1} \mathrm{~h}^{-1}$ via infusion pumps. Pneumoperitoneum was created by intraperitoneal insufflation of $\mathrm{CO}_{2}$ with an insufflation pressure of $13-15 \mathrm{mmHg}$ and patient in the supine position. Patients were then placed in the steep Trendelenburg position ( $30^{\circ}$ from horizontal). Intraperitoneal pressure was maintained at $15 \mathrm{mmHg}$ during the induced pneumoperitoneum. A routine anaesthesia monitoring was performed on all patients (Table 1).

During anaesthesia all patients received warm venous infusion of saline solution $(0.9 \% \mathrm{NaCl}) 3 \mathrm{ml} \mathrm{Kg}^{-1} \mathrm{~h}^{-1}$ and thermal mattresses. Systolic arterial pressure was maintained at $100 \mathrm{~mm} \mathrm{Hg}$ or $70 \%$ of the preoperative value. Hypotension was treated with crystalloid fluid infusion or intravenous boluses of ephedrine.

After surgery the residual neuromuscular blockade was reversed with a mixture of atropine (Galenica Senese, Siena, Italy) $1.5 \mathrm{mg}$ and neostigmine (Intrastigmina ${ }^{\mathrm{TM}}$, 
Table 1 Clinical characteristics and peri-operative data of patients with prostate cancer who underwent surgery with TIVA-TCI or BAL anaesthesia

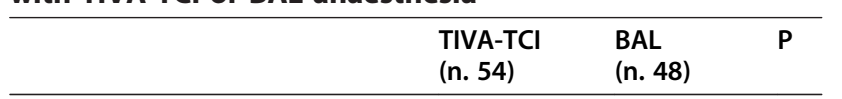

\section{Clinical data}

$\begin{array}{llll}\text { Age (yrs) } & 60.66(5.91) & 62.16(6.23) & 0.31 \\ \begin{array}{l}\text { Venous thromboembolism risk } \\ \quad \text { Highest risk }\end{array} & 54(100 \%) & 48(100 \%) & 1 \\ \begin{array}{lll}\text { Prostate cancer risk* } \\ \text { Intermediate-risk }\end{array} & & \\ \text { High-risk } & 26(48.1 \%) & 30(62.5 \%) & \\ & 28(51.8 \%) & 18(37.5 \%) & 0.17\end{array}$

ASA, $n$ (\%):

I

$\begin{array}{lll}4(7.4 \%) & 6(12.5 \%) & \\ 50(92.6 \%) & 42(87.5 \%) & 0.39\end{array}$

Histological grade of cancer

G2 (Gleason 5-6)

G3 (Gleason 7-10)

$15(27.8 \%) \quad 14(29.2)$

$39(72.2 \%) \quad 34(70.8 \%)$

pT, n (\%)

2

3

$\mathrm{pN}, \mathrm{n}(\%) \#$

0

1

Peri-operative data

Type of surgery

LRP

RALP

Time of anaesthesia $(\mathrm{min})$

Blood loss (ml)

Total amount of crystalloid received $(\mathrm{ml})$

Intra-operative body temperature

Intra-operative MAP (mmHg)

Intra-operative SpO2 (\%)

$32(66.7 \%)$

$24(44.4 \%)$

$16(33.3 \%)$

$17(85.0 \%) \quad 24(96.0 \%)$

0.20

$3(15.0 \%) \quad 1(4.0 \%)$

$36(66.7 \%) \quad 34(70.8 \%)-0.65$

$18(33.3 \%) \quad 14(29.2 \%)$

$107.5(16.8) \quad 101.4(26.2) \quad 0.26$

$123.3(131.1) \quad 121.4(110.6) \quad 0.81$

468.5 (110.21) $496.8(198.5) \quad 0.27$

$36.2(0.3) \quad 36.1(0.2) \quad 0.83$

$104.6(10.5) \quad 106.2(10.2) \quad 0.61$

$96.7(0.9) \quad 97.8(1.8) \quad 0.75$

Arterial lactate level ( $\mathrm{mmol} / \mathrm{l})$

\begin{tabular}{clll}
1 h post-surgery & $0.7(0.2)$ & $0.6(0.4)$ & 0.32 \\
24 h post-sugery & $1.7(0.2)$ & $1.8(0.2)$ & 0.82 \\
Intra-operative BE $(\mathbf{m m o l} / \mathbf{l})$ & $0.3(0.4)$ & $0.4(0.4)$ & 0.62 \\
Intra-operative $\mathbf{P a O 2}(\mathbf{m m H g})$ & $219.4(11.2)$ & $216.5(16.8)$ & 0.72 \\
\hline
\end{tabular}

Values are expressed in absolute values or mean (SD).

Abbreviations: TIVA-TCI total intravenous anaesthesia with target-controlled

infusion, $B A L$ balanced inhalation anaesthesia, $L R P$ conventional laparoscopic

radical prostatectomy, RALP robot-assisted laparoscopic prostatectomy.

*According to Guidelines on Prostate Cancer, European Association of

Urology, 2012.

\#Lymph node dissection was made in 45 out of 102 pts.
Lusofarmaco, Milano, Italy) $2.5 \mathrm{mg}$. Anaesthetic agents were switched off, and $100 \% \mathrm{O}_{2}$ was given with $8 \mathrm{l} \mathrm{min}$ fresh gas flow for $1 \mathrm{~min}$. In addition, a forced-air warming blanket was used post-surgery (Equator Covective Warming ${ }^{\mathrm{TM}}$, Smith Medical Italia, Milano, Italy).

After tracheal extubation all patients received ketoralac trometamina (Toradol, Recordati, Milano, Italy) $30 \mathrm{mg}$, ranitidine (Ranidil ${ }^{\mathrm{TM}}$, Menarini, Firenze, Italy) $50 \mathrm{mg}$ and morphine (Recordati) $2 \mathrm{mg}$ in bolus and then by a controlled analgesia device (Deltec ${ }^{\mathrm{TM}}$, Smiths Medical ASD, St Paul, MN).

\section{Clinical parameters}

The risk of venous thromboembolism was evaluated according to the model proposed by Caprini et al. [25] and Bergqvist et al. [26]. Patients were divided into 4 different levels of risk: low (score 0-1), moderate (score 2), high (score 3-4), highest (score $>4$ ). The following clinical parameters were also evaluated: (a) global assessment of anesthetic risk (ASA), (b) grading of prostate cancer (Gleason score), (c) pathological tumor-node-metastasis stage, (d) time of surgery, (e) quantity and type of liquids administered, (f) blood loss, (g) peri-operative complications such as hypertension, hyperglycemia, hypothermia, infections and pain (evaluated by a 6-point verbal rating scale: 0: no pain to 5: most severe pain imaginable).

In all patients, the presence of venous thrombosis by clinical observation, venous and pelvic ultrasound were evaluated in the peri-operative period and on days 8 and 21 after surgery.

\section{Prophylaxis anti-thrombosis}

Since in most of our patients changes in pro- and anticoagulant and fibrinolytic markers were observed in the peri-operative period, an anti-thrombotic prophylaxis was made 24 hrs post surgery, for 4 weeks, by using Enoxaparina (Clexane ${ }^{\mathrm{TM}}$, Sanofi-Aventis, Milano) $4000 \mathrm{UI} /$ die .

\section{Prothrombotic markers}

Before the induction of anaesthesia (T0), $1 \mathrm{hr}$ post-surgery (T1) and 24 hrs post-surgery (T2), the following factors were evaluated: (a) procoagulant markers: fibrinogen, TAT, F1 + 2 and FVIII; (b) fibrinolysis markers: PAI-1, D-dimer; (c) platelet-aggregating properties: p-selectin; (d) hemostatic system inhibitors: AT, protein $\mathrm{C}(\mathrm{PC})$ and protein S (PS) activity.

Blood samples were collected in tubes without additives containing 3.2\% sodium citrate (Vacutainer, BectonDickinson, Franklin Lakes, NJ USA). Samples were centrifuged within $1 \mathrm{~h}$ at $2500 \mathrm{~g}$ for $20 \mathrm{~min}$, to obtain platelet-poor plasma. The plasmas were immediately tested. Moreover, plasma and serum samples were separated and stored in multiple aliquots at $-80^{\circ} \mathrm{C}$ for subsequent testing. All coagulation parameters (PT, 
aPTT, fibrinogen, AT, D-dimer, PC, PS, FVIII) were assayed by clotting, chromogenic and immunological methods on fully-automated ACL TOP analyzer using HemosIL commercial kits (Instrumentation Laboratory Company, Bedford, MA USA). Abnormal values were defined by the clinical laboratory or manufacturer's assay. Plasma levels of TAT and F1 +2 were measured by enzyme-linked immunosorbent assay Enzygnost ${ }^{\circ}$ TAT micro and Enzygnost $^{\ominus} \mathrm{F} 1+2$ mono kits, respectively (Siemens Healthcare Diagnostics Inc, NY USA), according to the manufacturer's instructions. Both assays employ the quantitative sandwich enzyme immunoassay technique. All samples showing values above the standard curve were re-tested with appropriate dilutions. Plasma levels of PAI-1 were measured with the enzyme-linked immunosorbent assay Asserachrom ${ }^{\circ}$ kit (Diagnostica Stago, Asnieres, France), according to the manufacturer's instructions. Plasma p-selectina levels were determined by Human sP-Selectin enzyme immunoassay (R\&D Systems, Inc Minneapolis, MN USA), according to the manufacturer's instructions, employing the quantitative sandwich enzyme immunoassay technique.

\section{Statistical analysis}

Data were analyzed with Statistical Package for the Social Sciences (SPSS) 14.0 software. Continuous and categorical variables were expressed as the mean \pm standard deviation or standard error and as frequency values and proportions, respectively. Pearson's chi-square test was used to assess possible differences in dichotomous variables between the various groups examined. The means of normally distributed data were compared with the Student's $t$-test. In other cases, the groups were compared with the Mann-Whitney's $U$ test. $\mathrm{P}$ values of the tests were adjusted using the Bonferroni method. Paired samples were analyzed by $t$-test and Wilcoxon Signed Ranks Test. Multiple linear regression was used in order to test the effect of anaesthesia, surgery and clinical characteristics of patients on changes of prothrombotic markers $24 \mathrm{~h}$ post-surgery (T2 time). A p-value of $<0.05$ was considered statistically significant.

\section{Results}

\section{Clinical characteristics of the patients}

The clinical characteristics of the patients enrolled in the study are reported in Tables 1 and 2. No significant differences were observed regarding age between TIVA-TCI and BAL patients.

Thirty-two out of 102 patients (31.4\%) underwent RALP and were equally distributed between the TIVA-TCI and BAL. The lymph node dissection was made in 45 out of 102 pts (44.1\%).

All patients were at highest risk of venous thromboembolism, according to the model proposed by Caprini et al. [25] and Bergqvist et al. [26] (being all neoplastic and undergoing surgery); 10 of these (9.8\%) had an ASA I whereas $92(90.2 \%)$ an ASA II.

Thirty-nine patients of TIVA-TCI group (72.2\%) and 34 of BAL group (70.8\%) showed a high grade prostatic carcinoma (G3) with Gleason score $\geq 7$.

Patients undergoing LRP showed a locally more advanced tumor (pT3) as compared to those treated with RALP (Table 2). No significant differences were observed regarding lymph node involvement $(\mathrm{pN})$. The mean duration of anesthesia was $103.8 \pm 26.1 \mathrm{~min}$, with no differences between the TIVA-TCI and BAL groups $(\mathrm{p}=0.26)$.

During surgery a light decrease in hematocrit and hemoglobin concentration was observed in both groups, but intra-operative blood loss was similar. Also, the volume of crystalloid administered during anaesthesia was similar in both groups. Similarly, no statistical differences were observed regarding hemodynamic and respiratory parameters. None of the patients experienced adverse clinical events during their postoperative course.

In all patients no TED was observed in the postoperative period and in a 2-yr follow-up. This is probably due to the anti-thrombotic prophylaxis which was carried out for ethical reasons in all patients 24 hrs post surgery because intra-operative changes of some pro-coagulant markers were observed. Lymph node metastases were detected in only 4 out of 45 patients with lymph node dissection (8.9\%): one in the TIVA-TCI group and 3 in the BAL group $(\mathrm{p}=0.32)$.

\section{Types of anaesthesia and prothrombotic markers}

Changes of prothrombotic markers associated with the use of different techniques of anesthesia are reported in Tables 3 and 4 . No statistically significant differences were observed in the baseline values of biomarkers (at T0) between TIVA-TCI and BAL groups, even when we considered the type of surgery. In both TIVA-TCI and BAL patients a significant and continuous reduction in screen clotting time PT (given as percentage) was observed during post-surgery period (T2) as compared to T0 $(\mathrm{p}=0.001)$, while aPTT was shortened at T1 and then normalised on the first postoperative day (T2).

At the end of surgery (T1), both TIVA-TCI and BAL patients showed a marked and significant increase in pro-coagulant factors (TAT, F1 +2 and FVIII) and consequent reduction in haemostatic system inhibitors (AT, PC and PS) compared to the values measured prior to surgery ( $p \leq 0.004$ for each markers). The greatest increase was observed in the values of TAT and F1 + 2 (about 3 times compared to T0), while the values of FVIII increased approximately 30\%. F1 +2 and FVIII slightly reduced at $\mathrm{T} 2$ but remained significantly higher than basal levels ( $\mathrm{p} \leq 0.04$ for each markers). Only TAT values returned to pre-anaesthesia values. We observed 
Table 2 Clinical characteristics and peri-operative data of patients with prostate cancer, divided in 4 subgroups according type of anesthesia and surgery

\begin{tabular}{|c|c|c|c|c|c|}
\hline & TIVA-TCI LRP (n. 36 pts) & TIVA-TCI RALP (n. 18 pts) & BAL LRP (n. 34 pts) & BAL RALP (n. 14 pts) & $\mathbf{P}$ \\
\hline \multicolumn{6}{|l|}{ Clinical data } \\
\hline Age (yrs) & $61.4(5.7)$ & $59.5(6.7)$ & $63.2(5.8)$ & $60.1(7.7)$ & 0.25 \\
\hline \multicolumn{6}{|l|}{ ASA, n (\%): } \\
\hline I & $3(8.3 \%)$ & $1(5.6 \%)$ & $5(14.7 \%)$ & $1(7.1 \%)$ & 0.68 \\
\hline$\|$ & $33(91.7 \%)$ & $17(94.4 \%)$ & $29(85.3 \%)$ & $13(92.9 \%)$ & \\
\hline \multicolumn{6}{|l|}{ Histological grade of cancer } \\
\hline G2 (Gleason 5-6) & $9(25.0 \%)$ & $6(33.3 \%)$ & $10(29.4)$ & $4(28.6)$ & 0.93 \\
\hline G3 (Gleason 7-10) & $27(75.0 \%)$ & $12(66.7 \%)$ & $24(70.6 \%)$ & $10(71.4 \%)$ & \\
\hline \multicolumn{6}{|l|}{ pT, n (\%) } \\
\hline 2 & $12(33.3 \%)$ & $18(100 \%)$ & $18(52.9 \%)$ & $14(100 \%)$ & 0.001 \\
\hline 3 & $24(66.7 \%)$ & 0 & $16(47.1 \%)$ & 0 & \\
\hline \multicolumn{6}{|l|}{$\mathrm{pN}, \mathrm{n}(\%)^{*}$} \\
\hline 0 & $11(84.6 \%)$ & $6(85.7 \%)$ & $14(93.3 \%)$ & $10(100 \%)$ & 0.57 \\
\hline 1 & $2(15.4 \%)$ & $1(14.3 \%)$ & $1(6.7 \%)$ & 0 & \\
\hline \multicolumn{6}{|l|}{ Perioperative data } \\
\hline Time of anaesthesia (min) & $104.0(21.3)$ & $109.7(24.4)$ & $98.8(30.2)$ & $105.2(24.8)$ & 0.32 \\
\hline Blood loss (ml) & $119.2(140.3)$ & $128.3(150.1)$ & $118.2(121.4)$ & $125.2(131.5)$ & 0.30 \\
\hline $\begin{array}{l}\text { Total amount of crystalloid } \\
\text { received }(\mathrm{ml})\end{array}$ & $475.4(100.4)$ & $460.8(118.4)$ & $486.1(166.4)$ & $499.8(200.2)$ & 0.21 \\
\hline Intra-operative body temperature & $36.2(0.3)$ & $36.1(0.4)$ & $36.1(0.2)$ & $36.1(0.3)$ & 0.87 \\
\hline Intra-operative MAP (mmHg) & $103.8(11.8)$ & $105.3(12.5)$ & $105.4(12.4)$ & $106.8(12.2)$ & 0.54 \\
\hline Intra-operative SpO2 (\%) & $96.7(0.9)$ & $96.7(0.9)$ & $97.8(1.8)$ & $97.8(1.8)$ & 0.75 \\
\hline \multicolumn{6}{|l|}{ Arterial lactate level (mmol/l) } \\
\hline $1 \mathrm{~h}$ post-surgery & $0.7(0.2)$ & $0.7(0.3)$ & $0.6(0.3)$ & $0.6(0.4)$ & 0.81 \\
\hline $24 \mathrm{~h}$ post-sugery & $1.8(0.3)$ & $1.7(0.2)$ & $1.7(0.3)$ & $1.8(0.3)$ & 0.77 \\
\hline Intra-operative BE (mmol/l) & $0.3(0.4)$ & $0.4(0.3)$ & $0.3(0.4)$ & $0.4(0.3)$ & 0.78 \\
\hline Intra-operative $\mathrm{PaO} 2(\mathrm{mmHg})$ & $220.6(13.2)$ & $218.8(13.4)$ & $214.6(18.6)$ & $219.5(19.0)$ & 0.22 \\
\hline
\end{tabular}

Values are expressed in absolute values or mean (SD).

Abbreviations: TIVA-TCI total intravenous anaesthesia with target-controlled infusion, BAL balanced inhalation anaesthesia, $L R P$ laparoscopic radical prostatectomy, $R A L P$ robot-assisted laparoscopic prostatectomy.

*Lymph node dissection was made in 45 out of 102 pts.

a corresponding increase in anti-coagulant factors that remains significantly lower than prior to surgery $(\mathrm{p}=0.001)$.

Fibrinogen levels significantly decreased at $\mathrm{T} 1$ in comparison to the initial values, but rose significantly 24 hours post-surgery in both groups, showing an increase of about $20-30 \%$ as compared to T0 values $(\mathrm{p}=0.001)$.

Changes in pro-coagulant factors and haemostatic system inhibitors were similar in both TIVA-TCI and BAL patients with no significant differences between the two groups of patients. In regards to the fibrinolysis system, D-dimer concentration in TIVA-TCI group, levels increased about 6-fold at T1 compared to baseline level $(\mathrm{p}=0.001$, Table 3$)$, while in BAL patients it showed an increase of about 4-fold ( $p=0.001$, Table 4). Both groups showed a decrease of D-dimer at T2 even if the concentration remained higher than baseline levels $(\mathrm{p}=$ 0.001), with no significant differences between TIVA-TCI and BAL patients.

Levels of the PAI-1, the principal inhibitor of the fibrinolysis system, and D-dimer remained constant between $\mathrm{T} 0$ and $\mathrm{T} 1$ but significantly increased at $\mathrm{T} 2$ in both groups.

Grading of prostate cancer evaluated by Gleason score and pathological tumor stages showed no significant effects on changes in prothrombotic markers observed both in the TIVA and BAL groups. Similarly, it was observed for all other clinical parameters analyzed.

\section{Surgery and prothrombotic markers}

Multivariate analysis demonstrated that only p-selectin was significantly correlated to the type of anesthesia and 
Table 3 Changes of prothrombotic markers in patients with prostate cancer who underwent surgery with total intravenous anesthesia with target-controlled infusion (TIVA-TCI) before the induction of anaesthesia (T0), $1 \mathrm{hr}$ post-surgery (T1) and $24 \mathrm{hrs}$ post-surgery (T2)

\begin{tabular}{|c|c|c|c|c|c|c|}
\hline & \multirow[t]{2}{*}{ T0 } & \multirow[t]{2}{*}{$\mathrm{T} 1$} & \multirow[t]{2}{*}{$\mathrm{T} 2$} & \multicolumn{3}{|c|}{$\mathbf{P}$} \\
\hline & & & & T0 vs T1 & T1 vs T2 & T0 vs T2 \\
\hline \multicolumn{7}{|l|}{ Screen clotting time } \\
\hline - PT (\%) & $93.1(1.3)$ & $85.6(1.2)$ & $82.5(1.2)$ & 0.001 & 0.21 & 0.001 \\
\hline - PTT (sec) & $29.6(0.6)$ & $26.8(0.7)$ & $27.6(0.8)$ & 0.003 & 0.07 & 0.18 \\
\hline \multicolumn{7}{|l|}{ Procoagulant markers } \\
\hline - Fibrinogen (mg/dL) & $285.5(7.1)$ & $262.3(6.6)$ & $353.3(8.8)$ & 0.004 & 0.001 & 0.001 \\
\hline - TAT (ng/L) & $9.1(1.9)$ & $22.8(3.2)$ & $9.7(2.4)$ & 0.002 & 0.004 & 0.79 \\
\hline$-\mathrm{F} 1+2(\mathrm{pmol} / \mathrm{L})$ & $210.8(27.3)$ & $622.1(64.2)$ & $364.4(45.6)$ & 0.001 & 0.001 & 0.007 \\
\hline - FVIII (\%) & $142.9(8.1)$ & $194.2(9.3)$ & $162.3(5.6)$ & 0.001 & 0.004 & 0.04 \\
\hline \multicolumn{7}{|l|}{ Fibrinolysis markers } \\
\hline - PAl-1 (ng/ml) & $15.2(1.4)$ & $21.9(5.8)$ & $36.1(9.8)$ & 0.41 & 0.20 & 0.04 \\
\hline - D-dimer ( $\mu \mathrm{g} / \mathrm{L})$ & $127.1(12.8)$ & $721.4(170.4)$ & $364.2(28.3)$ & 0.001 & 0.02 & 0.001 \\
\hline \multicolumn{7}{|c|}{ Haemostatic system inhibitors } \\
\hline - AT (\%) & $102.1(1.8)$ & $90.6(1.9)$ & $87.4(2.4)$ & 0.001 & 0.38 & 0.001 \\
\hline - protein C (\%) & $109.6(2.8)$ & $95.4(2.8)$ & $87.8(2.8)$ & 0.004 & 0.03 & 0.001 \\
\hline - protein S (\%) & $93.8(3.1)$ & $84.2(2.8)$ & $82.4(2.4)$ & 0.01 & 0.56 & 0.001 \\
\hline \multicolumn{7}{|c|}{ Platelet-aggregating properties } \\
\hline - p-selectin (ng/ml) & $37.9(2.0)$ & $36.8(2.4)$ & $33.5(2.6)$ & 0.78 & 0.37 & 0.28 \\
\hline
\end{tabular}

Values are mean (SD).

surgery $(\mathrm{p}=0.01)$. It is very important to note that the TIVA-TCI patients undergoing LRP showed a significant reduction in p-selectin levels between $\mathrm{T} 0$ and $\mathrm{T} 2$ $(p=0.001)$ while no changes were observed in the BAL group that did not use the robotic device (Figure 3). In contrast, a significant increase of p-selectin value was observed in patients undergoing RALP, regardless of the type of anesthesia, both 1 and 24 hours after surgery.

Patients undergoing RALP showed also $24 \mathrm{hrs}$ after surgery (T2), at univariate analysis, a greater reduction of PS, an inhibitor of haemostatic system, as compared to patients undergoing LRP $(\mathrm{p}=0.02)$ independent of the type of anaesthesia applied.

\section{Discussion}

Results of our study have demonstrated that both anaesthetic techniques seem to increase the risk of TED in prostate cancer patients undergoing LRP, mainly when the robot device was utilized, suggesting, therefore, the utility of a peri-operative thromboembolic prophylaxis. In fact, both TIVA-TCI and BAL patients showed a marked and significant increase in pro-coagulant factors and consequent reduction in haemostatic system inhibitors in the early post operative period ( $p \leq 0.004$ for each markers). However, this effect could be linked also to surgical stress, although the latter seems to have an independent effect only for p-selectin, as demonstrated by multivariate analysis. Moreover, the significant reduction of p-selectin levels between T0 and T2 (p = 0.001) observed in TIVA patients undergoing LRP, although this group of patients was composed mainly of patients at high-risk prostate cancer (as reported in Table 1), demonstrated that general anaesthetic agents used for TIVA have a better protective effect on the platelet activation in this subgroup of patients.

The evaluation of markers detecting activation of the hemostatic system represents a more sensitive way to assess the risk of thromboembolism as compared to the clinical assessment of TED. In our study, the activation of haemostatic system associated with thromboembolic risk was estimated by measuring levels of thrombin activation markers. TAT, PF1 +2 and FVIII increased in the immediate post operative period and gradually returned to near baseline levels. The peri-operative activation of coagulation also caused an increased of peri-operative PAI-1 levels, a potent inhibitor of fibrinolysis. The activation state persists during surgery and is independent of the anaesthetic agents used. These results confirm previous studies performed on patients undergoing major abdominal surgery for colon-rectal cancer [27], hepatic cancer resection [28], pneumonectomy for lung cancer [29].

No studies had previously examined whether different intra-operative anaesthetic regimens (TIVA-TCI vs. BAL) could cause different intra-operative profiles of highly 
Table 4 Changes of prothrombotic markers in patients with prostate cancer who underwent surgery with balanced inhalation anaesthesia (BAL) before the induction of anaesthesia (T0), 1 hr post-surgery (T1) and 24 hrs post-surgery (T2)

\begin{tabular}{|c|c|c|c|c|c|c|}
\hline & \multirow[t]{2}{*}{ T0 } & \multirow[t]{2}{*}{$\mathrm{T} 1$} & \multirow[t]{2}{*}{$\mathrm{T} 2$} & \multicolumn{3}{|c|}{$\mathbf{P}$} \\
\hline & & & & T0 vs T1 & T1 vs T2 & TO vs T2 \\
\hline \multicolumn{7}{|c|}{ Screening clotting time } \\
\hline - PT (\%) & $91.4(1.4)$ & $86.8(1.6)$ & $81.8(1.4)$ & 0.007 & 0.02 & 0.001 \\
\hline - PTT (sec) & $30.1(0.4)$ & $26.2(0.7)$ & $28.3(0.6)$ & 0.001 & 0.02 & 0.01 \\
\hline \multicolumn{7}{|l|}{ Procoagulant markers } \\
\hline - Fibrinogen (mg/dL) & $318.5(8.6)$ & $301.3(10.9)$ & $372.4(11.2)$ & 0.21 & 0.001 & 0.001 \\
\hline - TAT (ng/L) & $6.2(0.8)$ & $19.2(3.1)$ & $6.7(0.8)$ & 0.002 & 0.002 & 0.42 \\
\hline$-\mathrm{F} 1+2(\mathrm{pmol} / \mathrm{L})$ & $182.4(11.8)$ & $558.1(65.6)$ & $266.8(19.2)$ & 0.001 & 0.001 & 0.001 \\
\hline - FVIII (\%) & $123.4(4.8)$ & $228.2(15.8)$ & $169.2(6.2)$ & 0.001 & 0.001 & 0.001 \\
\hline \multicolumn{7}{|l|}{ Fibrinolysis markers } \\
\hline - PAl-1 (ng/ml) & $14.1(1.4)$ & $21.7(15.8)$ & $22.6(2.4)$ & 0.16 & 0.86 & 0.002 \\
\hline - D-dimer ( $\mu \mathrm{g} / \mathrm{L})$ & $175.5(22.6)$ & $622.1(175.4)$ & $421.3(30.6)$ & 0.003 & 0.07 & 0.001 \\
\hline \multicolumn{7}{|c|}{ Haemostatic system inhibitors } \\
\hline - AT (\%) & $97.8(1.7)$ & $92.0(1.7)$ & $89.1(1.8)$ & 0.04 & 0.25 & 0.001 \\
\hline - protein C (\%) & $105.2(3.8)$ & $99.3(2.7)$ & $88.5(2.7)$ & 0.18 & 0.03 & 0.001 \\
\hline - protein S (\%) & $95.6(2.4)$ & $91.2(2.4)$ & $81.8(2.6)$ & 0.08 & 0.01 & 0.001 \\
\hline \multicolumn{7}{|c|}{ Platelet-aggregating properties } \\
\hline - p-selectin (ng/ml) & $41.5(2.7)$ & $40.7(2.9)$ & $40.2(2.8)$ & 0.65 & 0.88 & 0.18 \\
\hline
\end{tabular}

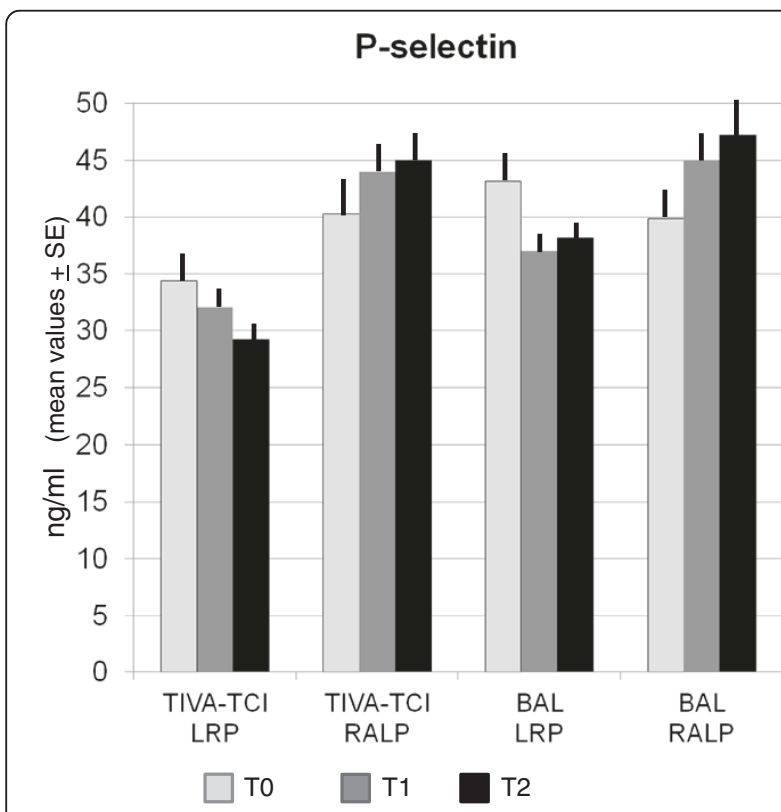

Figure 3 Changes of p-selectin levels between T0 (before the induction of anaesthesia) and T2 (24 hrs post-surgery) in patients undergoing conventional laparoscopic radical prostatectomy (LRP) or robot-assisted laparoscopic prostatectomy (RALP). TIVA-TCI patients undergoing LRP showed a significant reduction in $p$-selectin levels between T0 and T2 $(p=0.001)$ while no changes were observed in the BAL group. In contrast, a significant increase of p-selectin value was observed 24 hours after surgery (T2) in patients undergoing RALP, regardless of the type of anaesthesia. sensitive and specific coagulation and fibrinolysis markers in prostate cancer patients undergoing a highly standardized type of surgery (LRP or RALP). In this context, the results of our study seem to provide useful information in reducing the peri-operative trombo-embolic risk and improving the prognosis of cancer patients undergoing LRP and RALP.

Even though cancer patients who undergo surgery are targeted for thromboprophylaxis, widespread use of prophylaxis could determine the risk of intra-operative bleeding $[23,24]$ and a detrimental effect rather than a benefit. This problem is evident in prostate cancer patients undergoing surgery, especially in view of the increasingly frequent use of the robotic technique that has resulted in a significant reduction of surgical complications [30,31]. Although the American and European guidelines recommend prophylaxis in patients with prostate cancer [18-22], its use is currently widely debated given the different incidence of TED observed by several authors. A multicentric analysis of a number of institutions from both Europe and the United States showed a very low incidence of TED (about 0.5\%) [32]. A similar incidence (0.9\%) was reported from the California Cancer Registry [4]. Conversely, Osborne et al. [14] consider patients with prostate cancer at intermediate risk of TED similar to patients with uterine, rectal, colon and liver cancer.

Prostatectomy significantly increases the incidence of TED up to $2.9 \%$ and $3.9 \%$, as reported by $\mathrm{Hu} \mathrm{JC}$ et al. 
[17], irrespective of the surgical approach. Tewari et al. [33] in a recent meta-analysis on 400 original research articles on surgical treatment for prostate cancer and its complications reported that the rate of deep vein thrombosis was significantly lowest for RALP $(0.3 \%)$, intermediate for LRP (0.5\%) and highest for open surgery (1.0\%). More recently, Van Hemelrijck et al. [16] analysed thromboembolic events following prostatectomy in about 45.000 men collected in the Prostate Cancer Database Sweden. Risk of venous thromboembolism and pulmonary embolism occurred especially in the first 2 months after surgery with the highest risk in patients undergoing open or laparoscopic surgery with pelvic lymph node dissection while laparoscopic procedures without lymph node dissection were at lowest risk. Unfortunately, in this study authors did not created separate categories for LRP and RALP as the majority of laparoscopic surgery was performed with robotic assistance. In our case series, dissection of pelvic lymph node was not an independent risk factor for TED because no significant differences were demonstrated in the values of the markers analyzed among the various subgroups of patients studied. Moreover, it should be noted that in previous studies only the clinical incidence of venous thromboembolism was measured, but not the changes of coagulation factors. In other studies many biomarkers were specifically checked for their capacity to predict venous thromboembolism during the course of cancer disease [10], but changes in these markers due to different types of surgery, such as LRP or RALP, were not evaluated. Our results are even more surprising when we consider that the anesthetic drugs used both in TIVATCI and BAL, in particular propofol [34] and sevoflurane [35], act by inhibiting the platelet aggregation, although with different mechanisms.

Patients underwent RALP, compared to LRP group, showed a greater reduction of inhibitors of haemostatic system, such as protein $S$, and the increase of p-selectin, a cell adhesion molecule on the surface of activated endothelial cells and activated platelets [13]. Data present in the literature regarding the different risk of thrombosis in patients submitted to LRP or RALP are very few. In a recent study Saily et al. [36] observed that RALP activates coagulation, and thromboprophylaxis for high-risk patients even after minimally invasive surgery may be beneficial. In particular, patients undergoing RALP showed postoperatively increased levels of fibrinogen, factor VIII, d-dimer associated to a thrombocytosis, reflecting a coagulation activity. The greater risk of thrombosis with the RALP could be also related to the surgical stress that leads RALP to a major release of inflammatory mediators [37] or a greater oxidative stress induced by ischemia-reperfusion [38], determining the endothelial dysfunction and hypercoagulability [27]. This hypothesis is outlined by the fact that no differences were observed in other factors that may cause an activation of the haemostatic system in the peri-operative period such as anemia, hypoxia, hypothermia, hemodilution, hypotension, peritoneal insufflation, and Trendelenburg position $[39,40]$. We do not know whether changes in pro-coagulant factors may determine the occurrence of thrombotic complications since an anti-thrombotic prophylaxis was administered for ethical reasons $24 \mathrm{hrs}$ after surgery.

Our results suggest the use of a prophylaxis in all patients undergoing laparoscopic prostatectomy, in particular RALP, regardless of the type of anesthesia. Prophylaxis could not be required only in patients undergoing LRP with TIVA-TCI anaesthesia since a significant reduction in $\mathrm{p}$-selectin levels between T0 and T2 $(\mathrm{p}=0.001)$ was observed in this subgroup of patients. On the contrary, p-selectin did not change in patients undergoing LRP with BAL. Thus, the results we obtained suggest a greater inhibition effect of propofol, as compared to sevofluorane, on platelet aggregation pselectin mediated. The different effect of propofol and sevofluorane on p-selectin levels observed in our study is in agreement with previous observations reporting that sevofluorane inhibits human platelet aggregation induced by weak antagonists such as adenosine diphosphate, but not by strong agonists like thrombin $[41,42]$. Propofol, on the contrary, inhibits platelet aggregation mediated by thrombin [43] that regulates also the expression of p-selectin on platelets.

\section{Conclusions}

The marked and significant increase in pro-coagulant factors and consequent reduction in haemostatic system inhibitors we observed in the early post operative period suggests that a peri-operative thromboprophylaxis may be beneficial in cancer patients undergoing laparoscopic radical prostatectomy especially when a robot-assistance is used.

\section{Abbreviations}

LRP: Laparoscopic prostatectomy; RALP: Robot-assisted laparoscopic prostatectomy; TIVA-TCl: Total intravenous anesthesia with target-controlled infusion; BAL: Balanced inhalation anaesthesia; TED: Thromboembolic disease; F1 + 2: Prothrombin fragment $1+2 ;$ TAT: Thrombin-antithrombin complexes; PAI-1: Plasminogen activator inhibitor type 1; FVIII: Factor VIII; AT: Antithrombin; PC: Protein C; PS: Protein S.

\section{Competing interests}

Sofra M, Antenucci A, Gallucci M, Mandoj C, Papalia R, Claroni C, Monteferrante I, Torregiani G, Gianaroli V, Sperduti I and Forastiere E: No interest declared.

\section{Authors' contributions}

MS and EF contributed to conception and design of the study, acquisition, analysis and interpretation of data. AA, MG, CM and IS worked on the acquisition, analysis and interpretation of data. RP, CC, IM, GT and VG contributed to acquisition of data. All Authors were involved in drafting the manuscript or revising it critically for important intellectual content and gave final approval of the version to be published. 


\section{Funding}

This work was supported by a grant from "Istituto Nazionale Tumori Regina Elena".

\section{Author details}

'Department of Anaesthesiology, Regina Elena, Roma National Cancer Institute, Via Elio Chianesi 53, Roma 00144, Italy. ${ }^{2}$ Clinical Pathology, Regina Elena, Roma National Cancer Institute, Rome, Italy. ${ }^{3}$ Department of Urology, Regina Elena, Roma National Cancer Institute, Rome, Italy. ${ }^{4}$ Division of Biostatistic, Regina Elena, Roma National Cancer Institute, Rome, Italy.

Received: 4 April 2014 Accepted: 15 July 2014

Published: 17 August 2014

\section{References}

1. Sorensen HT, Mellemkjaer L, Olsen JH, Baron JA: Prognosis of cancers associated with venous thromboembolism. N Engl J Med 2000, 343:1846-50.

2. Prandoni P, Falanga A, Piccioli A: Cancer and venous thromboembolism. Lancet Oncol 2005, 6:401-10.

3. Heit JA: Venous thromboembolism: disease burden, outcomes and risk factors. J Thromb Haemost 2005, 3:1611-7.

4. Chew HK, Wun T, Harvey D, Zhou H, White RH: Incidence of venous thromboembolism and its effect on survival among patients with common cancers. Arch Intern Med 2006, 166:458-64.

5. ten Cate H, Falanga A: Overview of the postulated mechanisms linking cancer and thrombosis. Pathophysiol Haemost Thromb 2008, 36:122-30.

6. Heit JA, Silverstein MD, Mohr DN, Petterson TM, O'Fallon WM, Melton $\sqcup$ 3rd: Risk factors for deep vein thrombosis and pulmonary embolism: a population-based case-control study. Arch Intern Med 2000, 160:809-15.

7. Falanga A, Panova-Noeva M, Russo L: Procoagulant mechanisms in tumour cells. Best Pract Res Clin Haematol 2009, 22:49-60.

8. Falanga A, Marchetti M, Vignoli A: Coagulation and cancer: biological and clinical aspects. J Thromb Haemost 2013, 11:223-33.

9. Nierodzik ML, Karpatkin S: Thrombin induces tumor growth, metastasis, and angiogenesis: evidence for a thrombin-regulated dormant tumor phenotype. Cancer Cell 2006, 10:355-62.

10. Pabinger I, Thaler J, Ay C: Biomarkers for prediction of venous thromboembolism in cancer. Blood 2013, 122:2011-8.

11. Pabinger I, Ay C: Biomarkers and venous thromboembolism. Arterioscler Thromb Vasc Biol 2009, 29:332-6.

12. Van Haren RM, Valle EJ, Thorson CM, Guarch GA, Jouria JM, Andrews DM Sleeman D, Levi JU, Livingstone AS, Proctor KG: Long-term coagulation changes after resection of thoracoabdominal malignancies. J Am Coll Surg 2014, 218:846-54.

13. Chen M, Geng JG: P-selectin mediates adhesion of leukocytes, platelets, and cancer cells in inflammation, thrombosis, and cancer growth and metastasis. Arch Immunol Ther Exp (Warsz) 2006, 54:75-84.

14. Osborne NH, Wakefield TW, Henke PK: Venous thromboembolism in cancer patients undergoing major surgery. Ann Surg Oncol 2008, 15:3567-78.

15. Van Hemelrijck M, Adolfsson J, Garmo H, Bill-Axelson A, Bratt O, Ingelsson E, Lambe M, Stattin P. Holmberg L: Risk of thromboembolic diseases in men with prostate cancer: results from the population-based PCBaSe Sweden. Lancet Oncol 2010, 11:450-8.

16. Van Hemelrijck M, Garmo H, Holmberg L, Bill-Axelson A, Carlsson S, Akre O, Stattin P, Adolfsson J: Thromboembolic events following surgery for prostate cancer. Eur Urol 2013, 63:354-63.

17. Hu JC, Gu X, Lipsitz SR, Barry MJ, D'Amico AV, Weinberg AC, Keating NL: Comparative effectiveness of minimally invasive vs open radical prostatectomy. JAMA 2009, 302:1557-64.

18. Mandala M, Falanga A, Roila F: Management of venous thromboembolism (VTE) in cancer patients: ESMO clinical practice guidelines. Ann Oncol 2011, 22(6):vi85-92.

19. Gould MK, Garcia DA, Wren SM, Karanicolas PJ, Arcelus JI, Heit JA, Samama CM: Prevention of VTE in nonorthopedic surgical patients: antithrombotic therapy and prevention of thrombosis, 9th ed: American college of chest physicians evidence-based clinical practice guidelines. Chest 2012, 141:e227S-77S.

20. Lyman GH, Khorana AA, Kuderer NM, Lee AY, Arcelus Jl, Balaban EP, Clarke JM, Flowers CR, Francis CW, Gates LE, Kakkar AK, Key NS, Levine MN, Liebman HA, Tempero MA, Wong SL, Prestrud AA, Falanga A: Venous thromboembolism prophylaxis and treatment in patients with cancer:
American society of clinical oncology clinical practice guideline update. J Clin Oncol 2013, 31:2189-204.

21. Geerts WH, Bergqvist D, Pineo GF, Heit JA, Samama CM, Lassen MR, Colwell CW: Prevention of venous thromboembolism: American college of chest physicians evidence-based clinical practice guidelines (8th edition). Chest 2008, 133:381S-453S.

22. Siragusa $S$, Armani U, Carpenedo M, Falanga A, Fulfaro F, Imberti D, Laurora $R_{\text {, }}$ Molinari AC, Prisco D, Silingardi M, Verso M, Visona A: Prevention of venous thromboembolism in patients with cancer: guidelines of the Italian society for haemostasis and thrombosis (SISET). Thromb Res 2012, 129:e171-6.

23. Baron TH, Kamath PS, McBane RD: Management of antithrombotic therapy in patients undergoing invasive procedures. N Engl J Med 2013, 368:2113-24.

24. Tafur AJ, Wysokinski WE, McBane RD, Wolny E, Sutkowska E, Litin SC, Daniels PR, Slusser JP, Hodge DO, Heit JA: Cancer effect on periprocedural thromboembolism and bleeding in anticoagulated patients. Ann Oncol 2012, 23:1998-2005.

25. Caprini JA, Arcelus Jl, Reyna JJ: Effective risk stratification of surgical and nonsurgical patients for venous thromboembolic disease. Semin Hematol 2001, 38:12-9

26. Bergqvist D, Caprini JA, Dotsenko O, Kakkar AK, Mishra RG, Wakefield TW: Venous thromboembolism and cancer. Curr Probl Surg 2007, 44:157-216.

27. Modrau II, Iversen LL, Thorlacius-Ussing OO: Hemostatic alterations in patients with benign and malignant colorectal disease during major abdominal surgery. Thromb Res 2001, 104:309-15.

28. Weinberg L, Scurrah N, Parker EC, Dauer R, Marshall J, McCall P, Story D, Smith C, MCNicol L: Markers of coagulation activation after hepatic resection for cancer: evidence of sustained upregulation of coagulation. Anaesth Intensive Care 2011, 39:847-53.

29. Swiniarska J, Zekanowska E, Dancewicz M, Bella M, Szczesny TJ, Kowalewski J: Pneumonectomy due to lung cancer results in a more pronounced activation of coagulation system than lobectomy. Eur J Cardiothorac Surg 2009, 36:1064-8

30. Tewari A, Grover S, Sooriakumaran P, Srivastava A, Rao S, Gupta A, Gray R, Leung $R$, Paduch DA: Nerve sparing can preserve orgasmic function in most men after robotic-assisted laparoscopic radical prostatectomy. BJU Int 2012, 109:596-602.

31. Srivastava A, Chopra S, Pham A, Sooriakumaran P, Durand M, Chughtai B, Gruschow S, Peyser A, Harneja N, Leung R, Lee R, Herman M, Robinson B, Shevchuk M, Tewari A: Effect of a risk-stratified grade of nerve-sparing technique on early return of continence after robot-assisted laparoscopic radical prostatectomy. Eur Urol 2013, 63:438-44.

32. Secin FP, Jiborn T, Bjartell AS, Fournier G, Salomon L, Abbou CC, Haber GP, Gill IS, Crocitto LE, Nelson RA, Cansino Alcaide JR, Martinez-Pineiro L, Cohen MS, Tuerk I, Schulman C, Gianduzzo T, Eden C, Baumgartner R, Smith JA, Entezari $K$, van Velthoven $R$, Janetschek G, Serio AM, Vickers AJ, Touijer K Guillonneau B: Multi-institutional study of symptomatic deep venous thrombosis and pulmonary embolism in prostate cancer patients undergoing laparoscopic or robot-assisted laparoscopic radical prostatectomy. Eur Urol 2008, 53:134-45.

33. Tewari A, Sooriakumaran P, Bloch DA, Seshadri-Kreaden U, Hebert AE, Wiklund $P$ : Positive surgical margin and perioperative complication rates of primary surgical treatments for prostate cancer: a systematic review and meta-analysis comparing retropubic, laparoscopic, and robotic prostatectomy. Eur Urol 2012, 62:1-15.

34. Kozek-Langenecker SA: The effects of drugs used in anaesthesia on platelet membrane receptors and on platelet function. Curr Drug Targets 2002, 3:247-58.

35. Hirakata H, Ushikubi F, Toda H, Nakamura K, Sai S, Urabe N, Hatano Y, Narumiya S, Mori K: Sevoflurane inhibits human platelet aggregation and thromboxane A2 formation, possibly by suppression of cyclooxygenase activity. Anesthesiology 1996, 85:1447-53.

36. Saily VM, Petas A, Joutsi-Korhonen L, Taari K, Lassila R, Rannikko AS: Dabigatran for thromboprophylaxis after robotic assisted laparoscopic prostatectomy: retrospective analysis of safety profile and effect on blood coagulation. Scand J Urol 2014, 48:153-159.

37. Caine GJ, Stonelake PS, Lip GY, Kehoe ST: The hypercoagulable state of malignancy: pathogenesis and current debate. Neoplasia 2002, 4:465-73.

38. Glantzounis GK, Tsimaris I, Tselepis AD, Thomas C, Galaris DA, Tsimoyiannis EC: Alterations in plasma oxidative stress markers after laparoscopic operations of the upper and lower abdomen. Angiology 2005, 56:459-65. 
39. Schmitges J, Trinh QD, Sun M, Abdollah F, Bianchi M, Budaus L, Salomon G, Schlomm T, Perrotte P, Shariat SF, Montorsi F, Menon M, Graefen M, Karakiewicz PI: Venous thromboembolism after radical prostatectomy: the effect of surgical caseload. BJU Int 2012, 110:828-33.

40. Nguyen NT, Cronan M, Braley S, Rivers R, Wolfe BM: Duplex ultrasound assessment of femoral venous flow during laparoscopic and open gastric bypass. Surg Endosc 2003, 17:285-90.

41. Nozuchi S, Mizobe T, Aoki H, Hiramatsu N, Kageyama K, Amaya F, Uemura K, Fujimiya T: Sevoflurane does not inhibit human platelet aggregation induced by thrombin. Anesthesiology 2000, 92:164-70.

42. Huang GS, Li CY, Hsu PC, Tsai CS, Lin TC, Wong CS: Sevoflurane anesthesia attenuates adenosine diphosphate-induced P-selectin expression and platelet-leukocyte conjugate formation. Anesth Analg 2004, 99:1121-6.

43. Vasileiou I, Xanthos T, Koudouna E, Perrea D, Klonaris C, Katsargyris A, Papadimitriou L: Propofol: a review of its non-anaesthetic effects. Eur J Pharmacol 2009, 605:1-8.

doi:10.1186/s13046-014-0063-z

Cite this article as: Sofra et al:: Perioperative changes in pro and anticoagulant factors in prostate cancer patients undergoing laparoscopic and robotic radical prostatectomy with different anaesthetic techniques. Journal of Experimental \& Clinical Cancer Research 2014 33:63.

\section{Submit your next manuscript to BioMed Central and take full advantage of:}

- Convenient online submission

- Thorough peer review

- No space constraints or color figure charges

- Immediate publication on acceptance

- Inclusion in PubMed, CAS, Scopus and Google Scholar

- Research which is freely available for redistribution 